\title{
Damage Localization as a Possible Precursor of Earthquake Rupture
}

\author{
H. L. LI, ${ }^{1}$ Y. L. BAI, ${ }^{1}$ M. F. XIA, ${ }^{1,2}$ F. J. KE ${ }^{1,3}$ and X. C. YIN ${ }^{1,4}$
}

\begin{abstract}
Based on the concepts of statistical mesoscopic damage mechanics, the rupture of a heterogeneous medium is investigated in terms of numerical simulations of a network model, subjected to simple shear loading. The heterogeneities are simulated by varying the sizes and fracture strains of the elements of the network. Progressive damage is governed by a damage field equation and a dynamic function of damage (DFD). From the damage field equation, a criterion for damage localization can be derived, and the DFD can be extracted from the simulations of the network. Importantly, the DFD intrinsically governs the damage localization. Both stress-free and periodic boundary conditions for the network are examined. It is found that damage localization may be the underlying mechanism of eventual rupture and thus could be used as a possible precursor of earthquake rupture.
\end{abstract}

Key words: Heterogeneous medium, rupture, statistical mesoscopic damage mechanics, dynamic function of damage, damage localization.

\section{Introduction}

Many efforts have been made in an attempt to extract a realistic picture of the mechanical nature of earthquake rupture from seismological, geological and other geophysical data. Investigations have been carried out from the viewpoint of fracture mechanics (RICE, 1980; EvANS, 1978), stick-slip rate-dependent fault models (Dieterich, 1978; RiCE and RuINA, 1983), and the dynamic of fault rupture (DAS and AKI, 1977). Especially recently, earthquake nucleation and the effects of heterogeneity under simple (unidirectional) shear loading were examined numerically (Dieterich, 1992; Kemeny and Hagaman, 1992). These data indicate that earthquake rupture may result from the progressive damage of microstructures with varying sizes and strengths within fault sections. Thus the

\footnotetext{
${ }^{1}$ State Key Laboratory of Non-linear Mechanics, Institute of Mechanics, Chinese Academy of Sciences, Beijing 100080, China. E-mail: lihl@Inm.imech.ac.cn; baiyl@Inm.imech.ac.cn

${ }^{2}$ Department of Physics, Peking University, Beijing 100871, China. E-mail: xiam@Inm.imech.ac.cn

${ }^{3}$ Department of Applied Physics, Beijing University of Aeronautics and Astronautics, Beijing 100083, China. E-mail: kefj@Inm.imech.ac.cn

${ }^{4}$ Center for Analysis and Prediction, China Seismological Bureau, Beijing 100036, China. E-mail: yinxc@btamail.net.cn
} 
statistical nature of the progressive damage of a fault, owing to the heterogeneous distribution of microdefects, offers us a real challenge.

Recently, statistical mesoscopic damage mechanics has been developed to describe the collective behavior of distributed microcracks and microvoids nucleated at the sites of the original defects in solids (BAI et al., 1991). Also a transscale (from meso- to macroscopic) analysis has been proposed to correlate the important microstructural effects and the macroscopic mechanical behavior of solids, especially rupture. It has been found that, as a precursor of brittle rupture, the population of microdamage tends to form macroscopically localized damage (BAI et al., 1998). This may provide an informative way to understand the abrupt rupture of a fault containing numerous defects.

In the present study, the basic concepts and theoretical formulation of the statistical mesoscopic damage mechanics are combined with the numerical simulation of a network model under simple shear loading to reveal the intrinsic mechanism governing the rupture of a heterogeneous medium. The network model contains elastic bar-elements (chains) with varying sizes and fracture strains resembling the heterogeneity. The eventual rupture in such a model consists of many breakings of chains nearly at the same time, after which the network cannot sustain further loading. The data of the numerical simulations are analyzed from the viewpoint of statistical mesoscopic damage mechanics. The important microstructural effects are included in a function referred to as the dynamic function of damage (DFD). It is found that the DFD may be an inherent factor which governs damage localization and the concentration of microfractures prior to the eventual rupture. Moreover, a criterion for the emergence of damage localization can be expressed by means of the DFD. This may provide a way to give an alarm before the rupture.

\section{Basic Formulation of Statistical Mesoscopic Damage Mechanics}

In order to deal with the statistical nature of the progressive failure of a fault, we need a quantitative model which can reveal the collective effects of nucleation and the growth of microdamages created at the sites of original defects. Subsequently a transscale analysis should be carried out to estimate to what extent this collective behavior will influence the macroscopic rupture.

\section{Evolution Equation of Microdamage}

The collective behavior of microdamages can be described by the following fundamental equation in the light of statistical mesoscopic damage mechanics (BAI et al., 1991) 


$$
\frac{\partial}{\partial} \frac{n}{T}+\sum_{i=1}^{I} \frac{\partial}{\partial} \frac{\left(n \cdot P_{i}\right)}{p_{i}}=n_{N}-n_{A},
$$

where $n$ is the number density of microdamage and $T$ is time. $p_{i}$ are the independent variables describing the state of microdamages. For example, $p_{i}$ can be the current size of microdamage $c$, and the macroscopic spatial coordinates $x$ of the element where the microdamages locate, etc. $P_{i}=\dot{p}_{i}$ are the rates of variables $p_{i} . n_{N}$ and $n_{A}$ are nucleation and healing rate densities of microdamage, respectively.

\section{Macroscopic Damage}

The number density of microdamages can be related to a macroscopic measurement of damage by the following definition.

$$
D=\int_{0}^{\infty} \psi n d c
$$

where $\psi$ is the average failure volume of a microdamage. When we assume that there is a spherical failure volume surrounding each microdamage, $\psi=(\pi / 6) c^{3}$.

\section{Dynamic Function of Damage (DFD)}

With the definition of equation (2) in mind, one can turn the equation of number density of microdamages $n$, equation (1), into the governing equation for the macroscopic damage field

$$
\frac{\partial D}{\partial T}+\nabla \cdot(D \vec{v})=f
$$

with the dynamic function of damage $f$ defined by

$$
f=\int_{0}^{\infty} n_{N} \psi d c+\int_{0}^{\infty}\left(n A \psi^{\prime}-n_{A} \psi\right) d c
$$

where $\psi^{\prime}=\partial \psi / \partial c$, and $\vec{v}$ is the macroscopic velocity of the element where the microdamages locate. One key point of the present theoretical formulation is that all mesoscopic dynamics of microdamage, such as nucleation and healing rate densities of microdamage $n_{N}$ and $n_{A}$, as well as the average growth rate of microdamage $A$ are all included in the dynamic function of damage (DFD). The DFD serves to bridge the gap between the collective effects of mesoscopic damage mechanisms and the evolution of the macroscopic continuum damage field.

From the left-hand side of equation (3), the dynamic function of damage may be expressed as a function of the macroscopic mechanical variables and damage $D$. Without loss of generality, the DFD is assumed to be a function of damage $D$ and far-field shear stress $\tau$ in the simple shear case $f=f(\tau, D)$ as we discuss hereafter in this paper. 


\section{Prediction of Damage Localization}

According to the idea that damage localization may occur beyond a threshold and lead to eventual rupture (Fig. 1), it is of critical interest to propose a criterion which corresponds to this threshold. With the derivation given in (BAI et al., 1998), here we only cite the expression for a simple lower bound of the criterion for damage localization. It is assumed that the damage localization would occur once the relative gradient of damage begins to increase with time. Under unidirectional shear loading, this is expressed as

$$
\frac{\partial}{\partial T}\left[\frac{\partial D}{\partial Y} / D\right]>0
$$

where $T$ and $Y$ are temporal and spatial coordinates, respectively. ( $Y$-direction is perpendicular to the section of damage localization.) Actually, it is

$$
\left(\frac{\partial\left(\frac{\partial D}{\partial Y}\right)}{\partial T}\right) /\left(\frac{\partial D}{\partial Y}\right)>\left(\frac{\partial D}{\partial T}\right) / D .
$$

Under quasi-static simple shear conditions, with the help of equation (3), the above inequality gives a simple lower bound to damage localization as

$$
f_{D}>\frac{f}{D}
$$

where $f_{D}=(\partial f / \partial D)$. It is noteworthy that the dynamic function of damage $f$ not only seems to characterize the evolution of collective microdamages, but also to intrinsically govern the damage localization process. This is why the DFD is emphasized in the analysis. In the next sections, we will show how these concepts and the criterion derived from the statistical mesoscopic damage mechanics can be
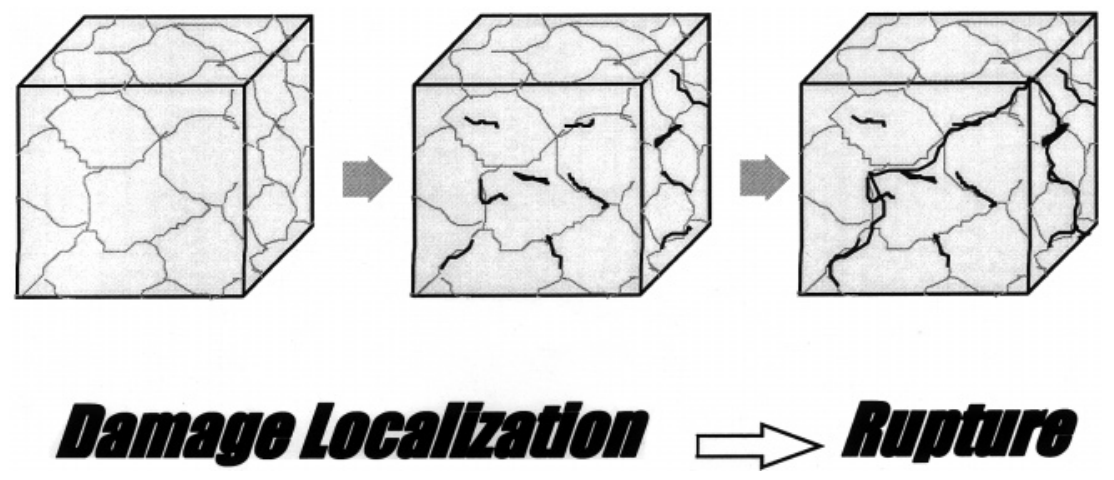

Figure 1

An illustration: damage localization leads to eventual rupture of solids. 
extracted from the numerical results when we use a network model under unidirectional shear stress to simulate the progressive failure of a fault section. We show how to predict the occurrence of damage localization prior to rupture, based on the data from numerical simulations.

\section{A Network Model}

In this section, we use a two-dimensional network model (XIA et al., 1996; LiANG et al., 1997) shown in Figure 2 to simulate the rupture process of a heterogeneous medium under simple shear loading. The network consists of bar-elements (chains). With increasing shear loading, the chains are stretched or contracted linear elastically until breaking, when their stretching strains exceed their fracture strains. Surmise that all chains have the same elastic modulus. Once some chains break, their stresses will be redistributed to their neighbors. This may induce further breaking or the network may attain a new quasi-equilibrium state. We presume that this would resemble the progressive degradation to eventual rupture of the heterogeneous medium. In order to simulate the heterogeneous medium, the chains are assumed to be randomly distributed in the network (see Fig. 2) with their lengths and fracture strains constrained by distribution functions (see Fig. 3).

The parameters adopted in the numerical simulations are as follows:

- The total number of bar-elements in each network sample is 4120 , and the total number of nodes is 1423 ;

- The fracture strains of chains are uniformly distributed between 0 and $2.0 \times$ $10^{-3}$, with the mean value being $10^{-3}$ (Fig. 3(a));

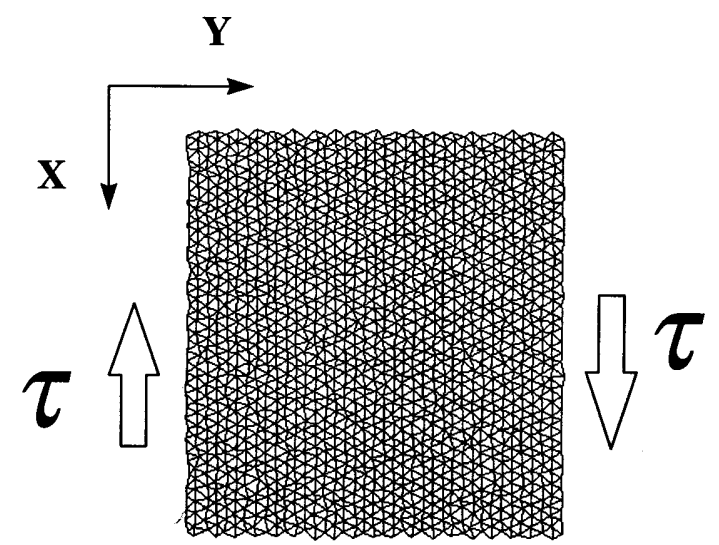

Figure 2

A sample of the network model under simple shear loading. 


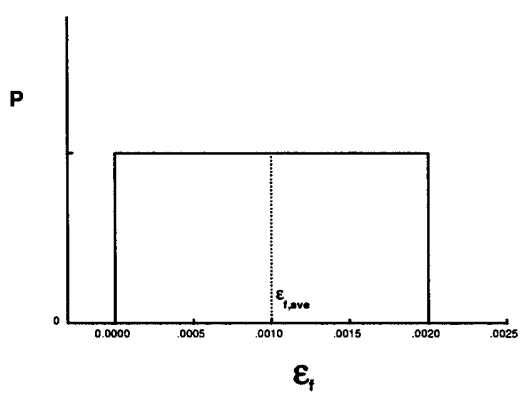

(a)

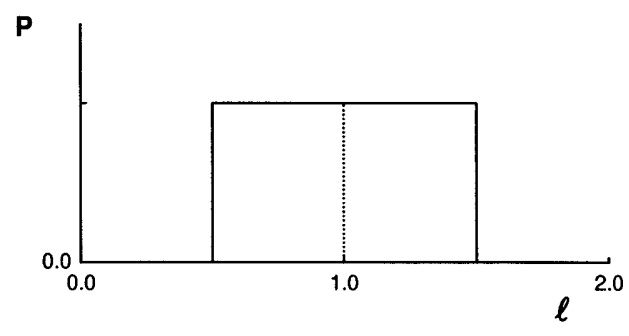

(b)

Figure 3

An uniform distribution of (a) fracture strains $\varepsilon_{f}$ and (b) lengths $\ell$ of chains ( $P=$ numbers of chains with $\varepsilon_{f}($ or $\ell$ )/the total of chains).

- The lengths of chains are uniformly distributed between 0.5 and 1.5, with a mean length of 1.0, the length scale (Fig. 3(b)).

At each step, we calculate the following quantities:

- Far-field stress $\tau$;

- The fraction of broken chains, as the damage $D$;

- The far-field displacement and the corresponding strain $\gamma$.

Here stress $\tau$ is dimensionless by dividing by the elastic modulus of chains.

\section{Numerical Results of the Network Model}

As described in the previous section, the parameters which represent the statistical features of chains in samples of network are specified. However, the samples of network are different from each other due to fluctuations in length and fracture strain of chains. As a result, the eventual rupture patterns and the value of peak stress for different samples may differ distinctly. We wonder whether general intrinsic characteristics, which govern the concentration of microfractures and the eventual rupture, can be captured for all these random samples.

From the obtained numerical data, the dynamic function of damage can be deduced. In fact, in one-dimensional approximation, equation (3) can be rewritten as follows

$$
f=\frac{\partial D}{\partial T}+\frac{D}{1+\theta} \dot{\theta},
$$

where $\theta$ is the volumetric strain. The above equation (8) suggests the feasibility of calculating the DFD $f$ inversely, if the data of macroscopic variables such as $\tau, D$ 


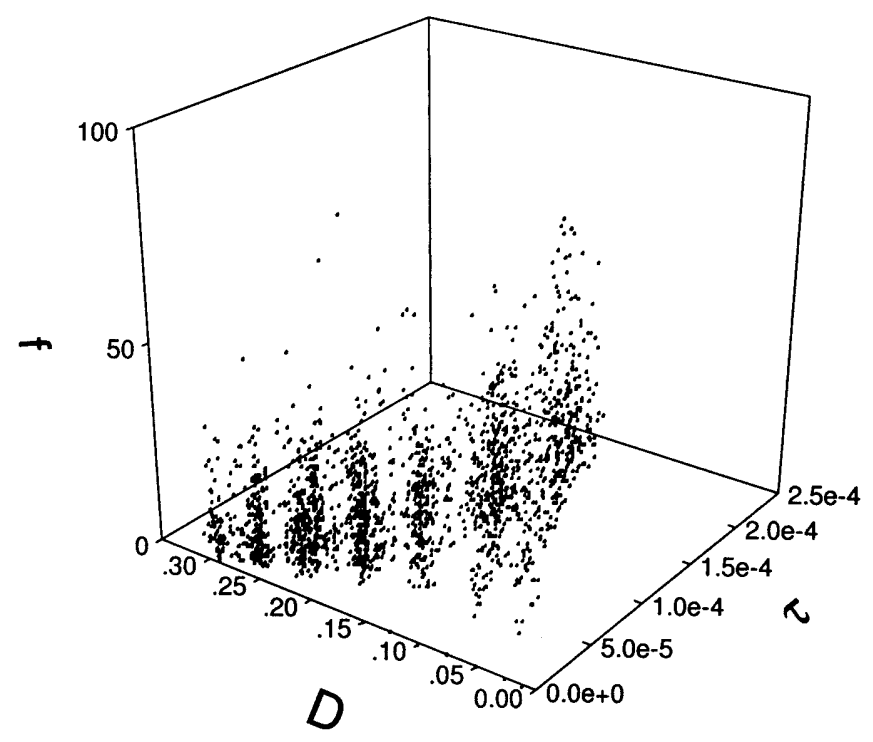

Figure 4

Dynamic function of damage (DFD) $f=f(D, \tau)$ under stress-free boundary conditions.

and $\theta$ can be obtained at different times. For example, these data can be obtained either from elaborately controlled experiments, seismological or geophysical observations, or as we do in this paper, from the numerical simulation. Then, we can deduce the DFD effectively according to equation (8). This provides a substitute for directly calculating the DFD from equation (4). Actually, the calculation of the DFD from equation (4) would involve the in situ experimental observation of the microdamage evolution (i.e., direct measurement of the nucleation rate $n_{N}$, growth rate $A$ and healing rate $n_{A}$ ). The complex mechanisms of microdamage evolution and their interaction may elude the direct estimate of the effects of nucleation, growth and coalescence, and thus, make the calculation of the DFD from equation (4) rather difficult, especially for heterogeneous media such as rocks.

\section{Free Boundary Conditions}

Thirty-five samples of the network model were run, and the DFD was calculated from equation (8) based on the data of $\tau, D$ and $\theta$ for all samples. All obtained data of DFD are plotted in the space $(f, D, \tau)$ in Figure 4. Now, we fit the data into the following expression

$$
f=a\left(1+c \cdot D+b \cdot e^{m \cdot D}\right) \sigma
$$

and obtain the fitting parameters $a=1.229 \times 10^{4}, b=0.2831, c=-15.31, m=$ 10.90 , and the relative deviation between the original and fitting values of $f$ is 
estimated to be 0.6563 . (The relative deviation $s=\sqrt{\sum_{i=1}^{N} d_{i}^{2} /(N-1)}$, where $d_{i}=$ $\left(f_{\text {original }}-f_{\text {fitting }}\right) / f_{\text {fitting, }}$, and $N$ is the total number of data.) This rather large relative deviation is reminiscent of the relative deviation of the fracture strains of network chains, $1 / \sqrt{3}$, a value in the same magnitude. Actually, the deviation of the calculated DFD indicates the existence of the sample-specific behavior of samples with heterogeneous microstructures. The dynamic evolution amplifies the differences between the samples as progressive damage of microstructure proceeds, and the deviation from the statistical average behavior becomes increasingly more significant. As a result, even samples with slight initial mesoscopic differences may exhibit significantly different failure behavior from sample to sample as the samples approach the eventual failure. This may explain the rather large deviation of the calculated DFD when the effects of sample-specific behavior are all included. However, a statistically average description may be assumed to properly characterize the process of damage accumulation before the emergence of damage localization when the deviation is relatively small. By fitting an expression to the calculated DFD data, we search for an approximate expression of the statistical average DFD which works well before the sample-specific behavior manifests itself.

Then, equation (9) is substituted into equation (7) to examine the occurrence of damage localization. This leads to the threshold of damage localization

$$
D_{c}=0.1530 \pm 0.0045
$$

(Even for the calculated DFD data including the effect of the most significant sample-specific behavior, we obtained a critical value $D_{c}=0.1575$.) What will really happen beyond this critical value?

We will make this point more clear by the run of other samples, and examine the validity of the criteria (7) and (10). Three additional samples (other than the 35 samples mentioned above) were run and their results are shown in Figures 5 (a)-(c). The critical "time" predicted by the criteria (7) and (10) is also illustrated by a cross in these figures. It can be seen that the failure patterns of the three samples are clearly different from each other due to fluctuations in microstructure. The stress-strain curves also differ in a distinct way. For one of the samples (Fig. 5(b)), a sharp stress drop such as a foreshock can be observed much earlier than the final rupture. However, for one of the other samples (Fig. 5(c)), the process between the damage localization predicted by criterion (10) and the final unstable rupture is extremely fast. However, the damage localization given by criterion (7) does provide an alarm ahead of eventual rupture for all three samples. In Figures 6(a)-(c), the evolution of damage patterns is shown. Thusly, we can identify minor concentration of microdamages in the network just after the predicted damage localization. More importantly, it is convincing that the criterion for damage localization based on the DFD data can serve as a precursor of abrupt rupture in random samples sufficiently well. This may provide evidence that the DFD intrinsi- 

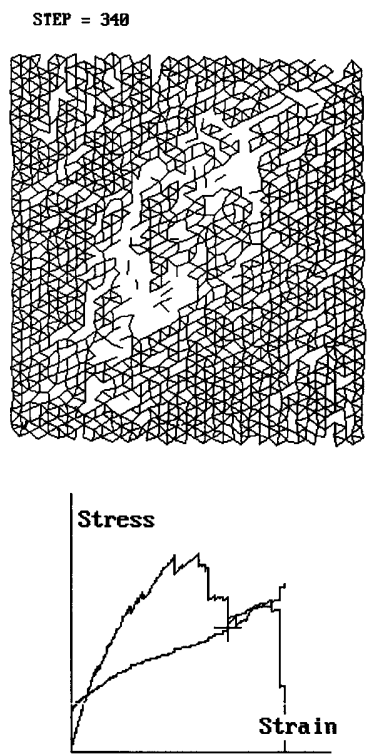

(a)
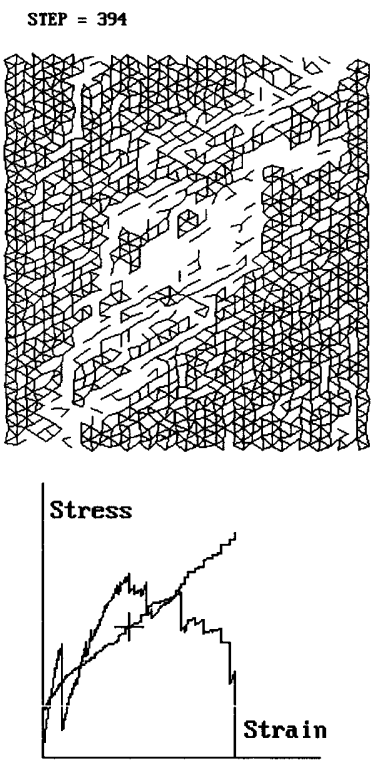

(b)
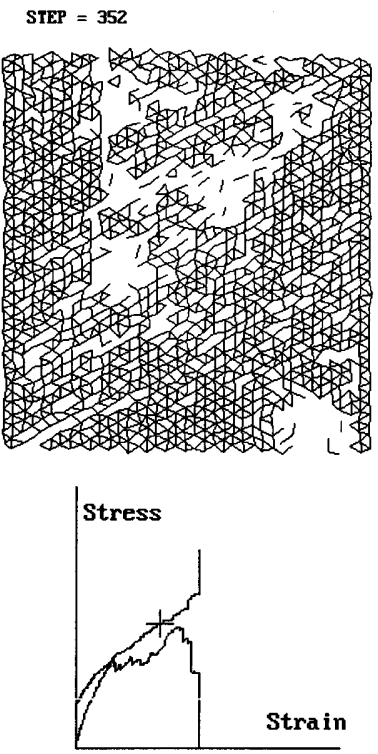

(c)

Figure 5

Rupture patterns and stress-strain curves of three samples, and the prediction given by the criterion for damage localization (marked by + ) under stress-free boundary conditions. The curve with + indicates the variation of damage $D$ with strain.

cally governs damage localization prior to rupture for all the samples, though their macroscopic mechanical behaviors appear to be decidedly different from each other.

\section{Periodic Boundary Conditions}

In order to approximately simulate the behavior of faults parallel to shear, periodic boundary conditions are also considered. In this case, the position and displacement of each node on the upper boundary in Figure 2 are assumed to be the same as that of its counterpart on the lower one.

Analogous to what we have done for the stress-free boundary case, the DFD data were obtained from simulations involving 5 random samples according to equation (8), and were shown in Figure 7. These data in the $(f, D, \tau)$ space are also fitted in formula (9) with $a=3558, b=0.9563, c=-22.18, m=6.449$, and the relative deviation between the original and fitting values of $f$ is 0.3184 .

The threshold value for damage localization is given by criterion (7) as

$$
D_{c}=0.1998 \pm 0.0043
$$



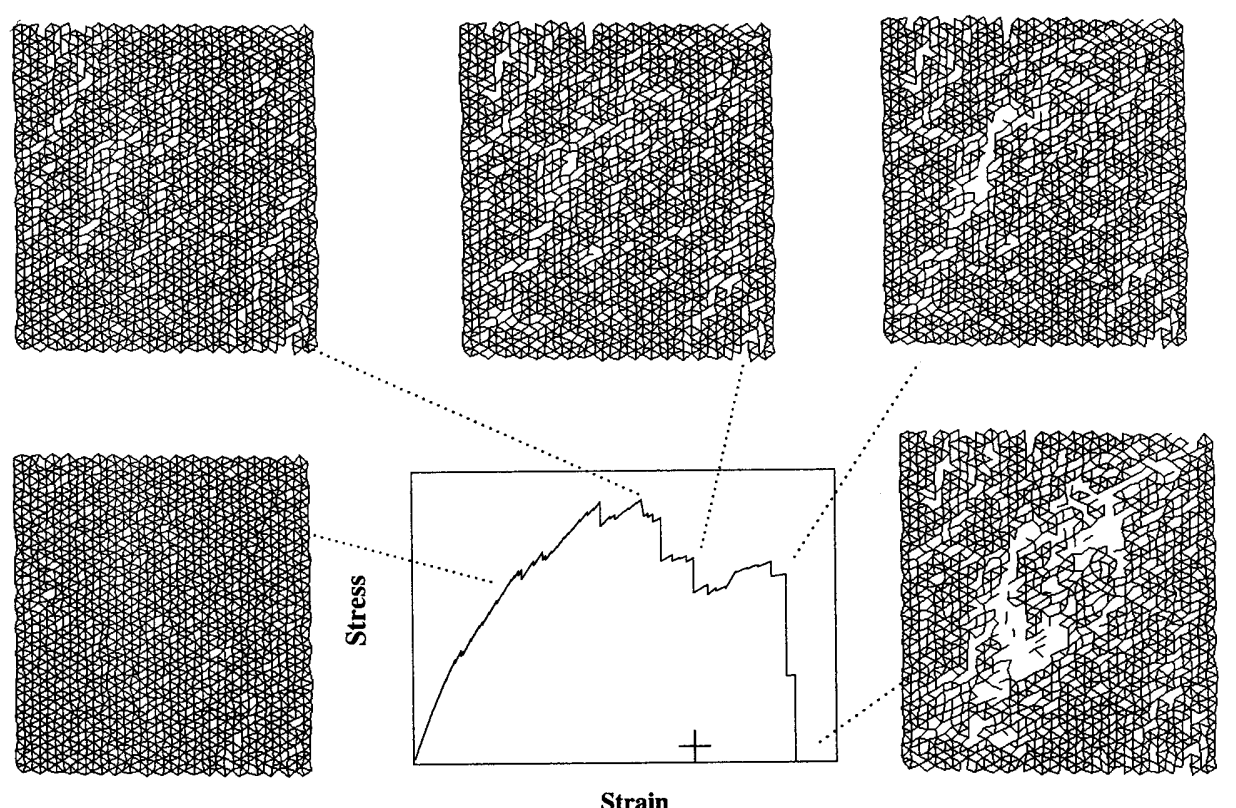

(a)
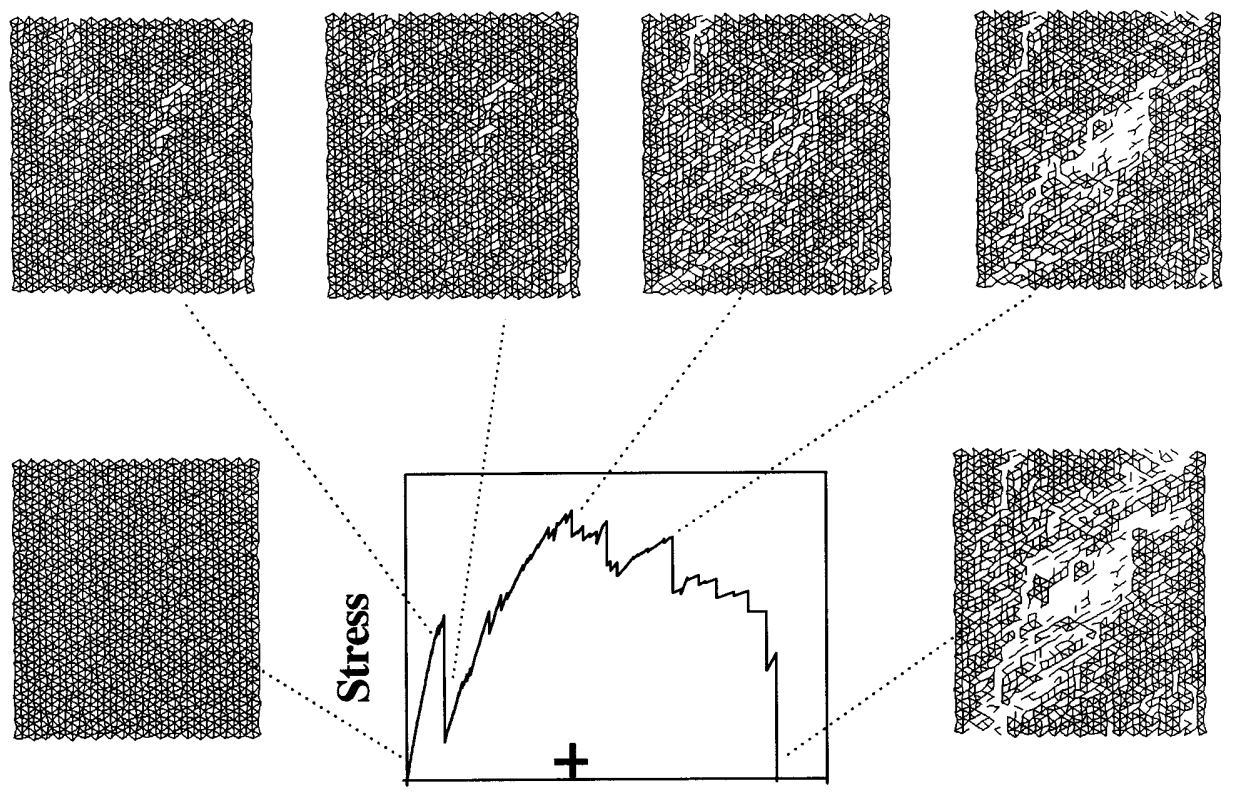

Strain

(b) 


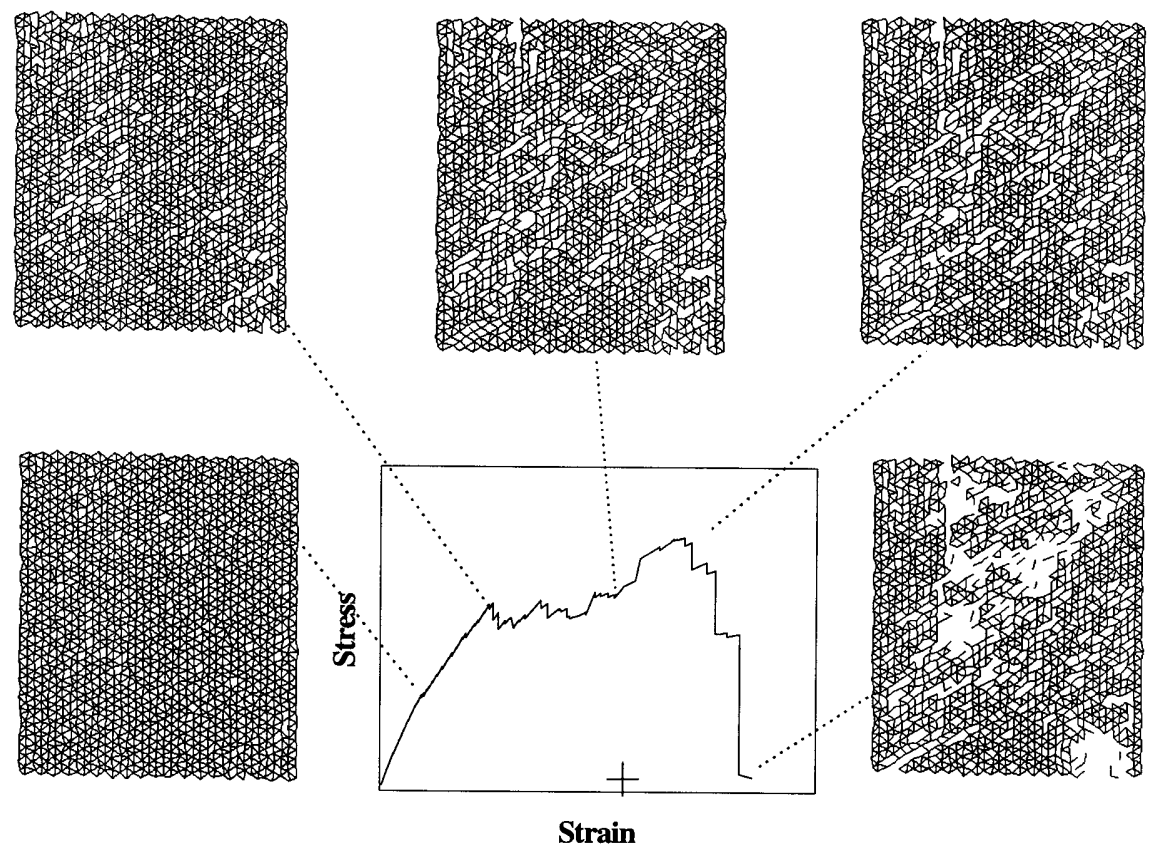

(c)

Figure 6

Damage patterns of the three samples at several times before and after predicted damage localization (marked by +). (a) Sample No. 1; (b) No. 2; (c) No. 3 (under stress-free boundary condition).

The rupture patterns of four other random samples are shown in Figures 8(a)-(d) with the stress-strain curves as well as the alarm given by equation (11). Figure 9 delineates the evolution of damage patterns of two samples with periodical boundary conditions. Compared to the stress-free boundary cases, the failure patterns of these samples are more likely to be parallel to the direction of shear stress. The threshold value of damage is larger than that of the stress-free boundary case. This indicates that the periodic constraint may retard the occurrence of damage localization. Nonetheless, with both stress-free and periodic boundary conditions, criterion (7) for damage localization always gives a proper alarm prior to the rupture.

The DFD, linking mesoscopic dynamics (nucleation, growth and coalescence) of microdamage and mesoscopic damage evolution, intrinsically characterizes the dynamic evolution of the heterogeneous damage (roughly speaking, the time rate of damage $D$ ). It is the dynamic evolution which amplifies the slight initial differences in microstructure, and results in the emergence of damage localization. Moreover, theoretical investigations (BAI et al., 1998) revealed that, for the same initial damage distribution, either homogeneous damage field or damage localization phenomenon may be observed, entirely depending on the expression of the DFD. 


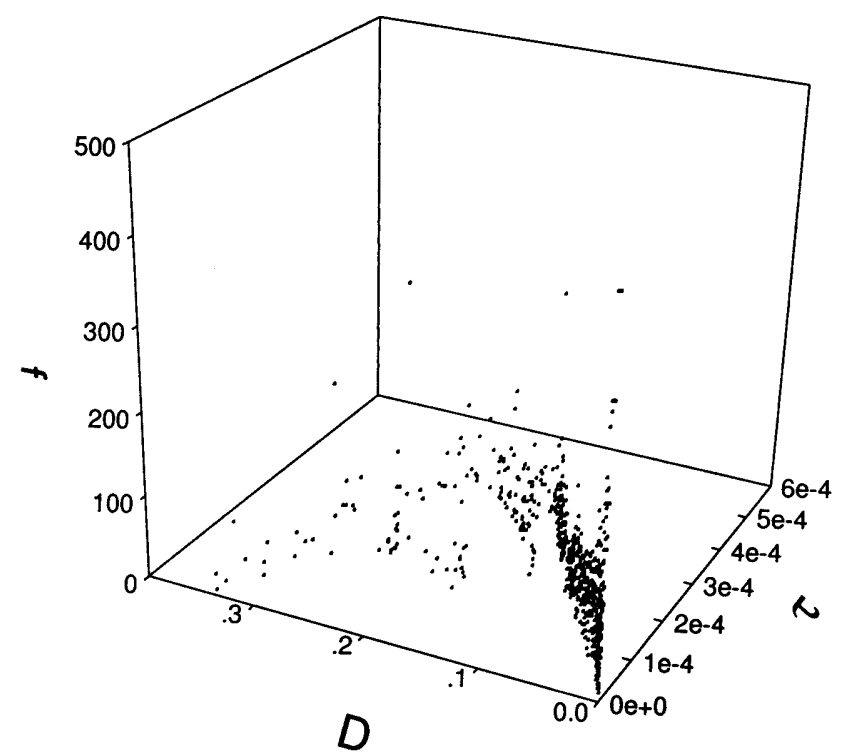

Figure 7

Dynamic function of damage (DFD) under periodic boundary conditions.
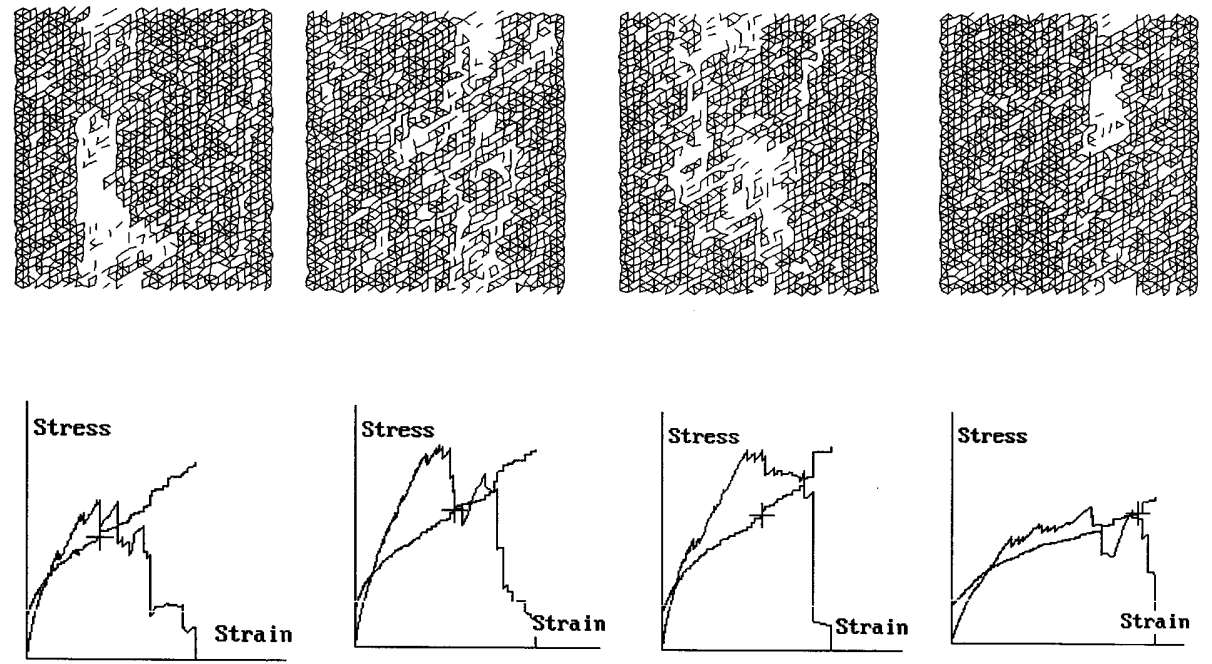

Figure 8

Rupture patterns and stress-strain curves of four samples, and the prediction given by the criterion for damage localization (marked by + ) under periodic boundary condition. The curve with + indicates the variation of damage $D$ with strain. 

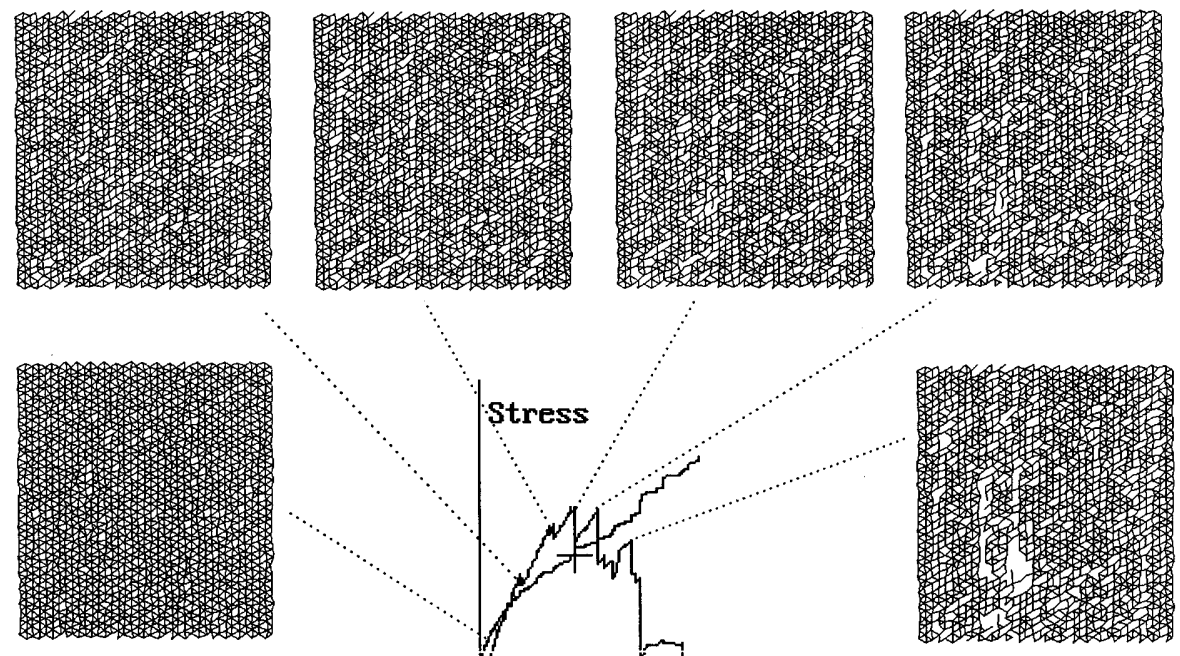

Strain

(a)
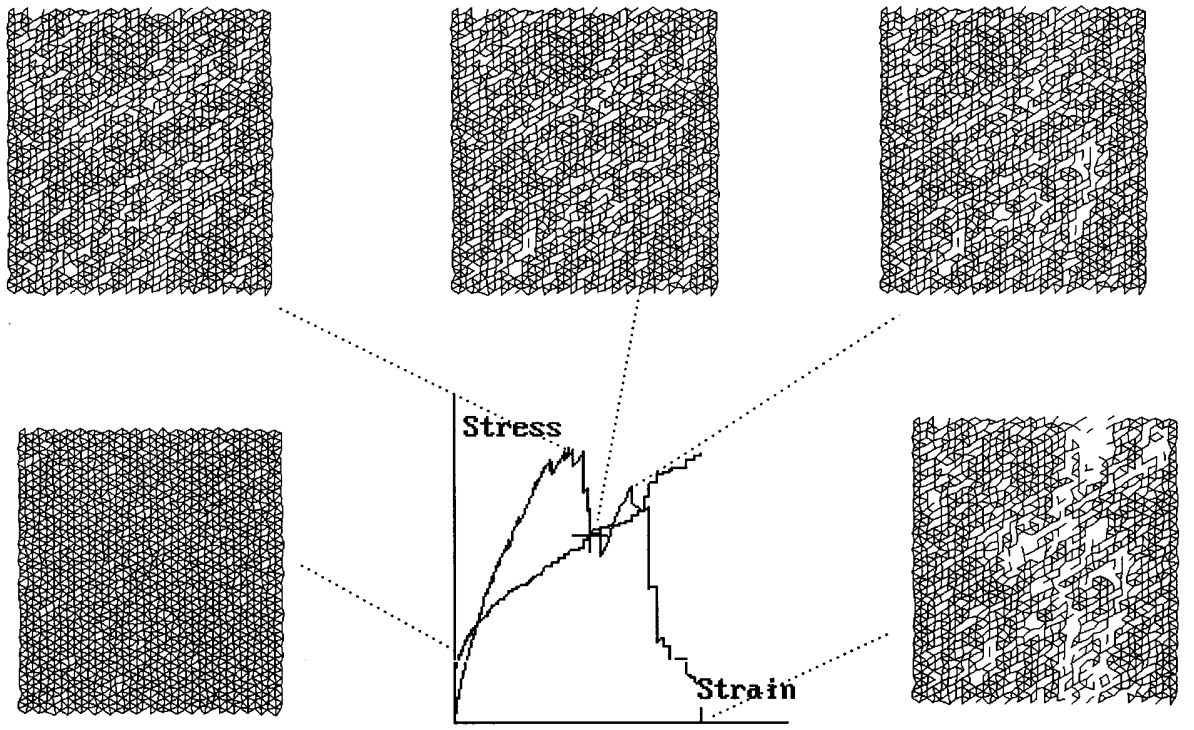

(b)

Figure 9

Damage patterns of the two samples at several times before and after predicted damage localization (marked by +). (a) Sample No. 1; (b) No. 2 (under periodic boundary condition) The curve with + indicates the variation of damage $D$ with strain. 
Thus, the DFD plays a key role in governing the occurrence of damage localization prior to eventual rupture.

\section{Conclusions}

The progressive damage of fault sections was investigated with numerical simulations from the viewpoint of statistical mesoscopic damage mechanics. The dynamic function of damage (DFD) introduced in the theoretical formulation is found to intrinsically govern the evolution of the heterogeneous damage field as well as damage localization prior to eventual rupture. With the information provided by the DFD, we may effectively predict the emergence of damage localization before the spatial concentration of microfractures can be observed. As a possible precursor for eventual rupture, the effectiveness of the criterion for damage localization based on the DFD is confirmed by the numerical results of the network model. Therefore damage localization may serve as a possible precursor of earthquake rupture.

\section{Acknowledgements}

This work is supported by National Natural Science Foundation of China (19891180, 19732060 and 19972004) and Chinese Academy of Sciences (KJ951-1201).

\section{REFERENCES}

BAI, Y. L., Ke, F. J., and XIA, M. F. (1991), Formulation of Statistical Evolution of Microcracks in Solids, Acta Mechanica Sinica 7, 59-66.

BAI, Y. L., XIA, M. F., Ke, F. J., and Li, H. L., Damage field equation and criterion for damage localization. In Rheology of Bodies with Defects (ed. Wang, R.) (Kluwer Academic Publishers 1998) pp. 55-66.

DAs, S., and AKI, K. (1977), Fault Planes with Barriers: A Versatile Earthquake Model, J. Geophys. Res. $82,5658-5670$.

DieTerich, J. (1978), Time Dependent Fraction and the Mechanics of Stick Slip, Pure appl. geophys. 116, $790-806$.

Dieterich, J. (1992), Earthquake Nucleation on Defects with Rate- and State-dependent Strength, Tectonophysics 211, 115-134.

Evans, A. G., Acoustic emission sources in brittle solids. In Fundamentals of Acoustic Emission (ed. Ono, K.) (Materials Dept., UCLA, Los Angeles 1978) pp. 209-227.

Kemeny, J. M., and Hagaman, R. M. (1992), An Asperity Model to Simulate Rupture along Heterogeneous Fault Surfaces, Pure appl. geophys. 138, 549-567.

LiAng, N. G., LiU, H. Q., and Xu, H. Q., A Multi-scale network model and parameter optimization of discontinuous fiber reinforced composites. In Macro/Micro/Meso Mechanical Properties of Materials (eds. Tokuda, M., and Bingye, XU) (Mie Academic Press 1997) pp. 269-275. 
RICE, J. R., The mechanics of earthquake rupture. In Physics of the Earth's Interior (eds. Dziewonski, A., and Boschi, E.) (North Holland, Amsterdam 1980) pp. 555-649.

Rice, J. R., and RuinA, A. L. (1983), Stability of Steady Fractional Slopping, J. Appl. Mech. 105, $343-349$.

XiA, M. F., Song, Z. Q., Xu, J. B., Zhao, K. H., and BAI, Y. L. (1996), Sample-specific Behavior in Failure Models of Disordered Media, Commun. Theor. Phys. 25, 49-54.

(Received July 22, 1999, revised February 1, 2000, accepted April 7, 2000) 